\title{
Fünfzig Jahre Verbraucherschutz
}

\author{
Ewoud Hondius
}

(C) VOe 2012

Am 15.03.2012 hielt der damalige amerikanische Präsident John Figerald Kennedy vor dem US-Kongress eine Rede, in der er für Verbraucher vier Grundrechte vorschlug: das Recht auf Sicherheit, das Recht auf Information, das Recht auf Wahlfreiheit und das Recht auf Gehör. In den Vereinigten Staaten von Amerika selbst hatte dieser Vorschlag keinen großen Erfolg, aber in Europa desto mehr. Zuerst war es der Europarat in Straßburg, der das Thema aufgriff, dann die Europäsche Union in Brüssel, die 1975 das erste Programm zum Schutze des Verbrauchers verabschiedete. In diesem Programm wurden fünf Verbrauchergrundrechte vorgeschlagen, die offenbar von der Rede Kennedy's inspiriert waren. Der Erolg war bemerkenswert: innerhalb von einigen Jahrzehnten verabschiedete die Europäische Union zahlreiche Richtlinien, die das Recht in Europa grundsätzlich geändert haben.

50 Jahre später, am 15.03.2012, wurde dieses Ereignis an der Universität Utrecht mit einer Konferenz über Vergangenheit („Die ersten fünfzig Jahre“) und Zukunft des Verbraucherrechts gefeiert. An der Organisation der Konferenz waren auch die Radboud Universität Nijmegen (Johan van der Gronden) und der Sozial-Ökonomische Rat (Thom van Mierlo) beteiligt.

Eine zentrale Frage, die auf der Konferenz diskutiert wurde, war die, ob das Verbraucherrecht innerhalb der nächsten 50 Jahre noch immer bestehen oder ob es im allgemeinen Privatrecht aufgenommen wird. Die letzte, von Susanne Augenhofer (Humboldt-Universität zu Berlin) vertretene These fand bei den Konferenzteilnehmern keinen Beifall.

Ewoud Hondius $(\square)$

Professor of European Private Law, University of Utrecht,

Molengraaff Instituut,

Janskerkhof 12, NL 3512 BL Utrecht, Netherlands

E-Mail: <e.h.hondius@uu.nl>
Zudem wurden mehrere aktuelle Einzelfragen des Verbraucherrechts erörtert.

Einige ausgewählte Konferenzbeiträge sind in diesem Heft veröffentlicht. Die Konferenz eröffnete Yvonne van Rooy (Kanzlerin der Universität Utrecht, Vorsitzende des Niederländischen Verbraucherbeirats und ehemalige Staatssekretärin). Ihr Eröffnungsvortrag über den Bedarf eines zufriedenstellenden Verbraucherschutzniveaus ist in diesem Heft ebenso aufgenommen wie die Überlegungen der USAmerikanerin Kathleen Gutman (Universität Antwerpen), die an der Konferenz aus persönlichen Gründen nicht teilnehmen konnte, über die Entwicklungen des US-amerikanischen Verbraucherrechts seit Kennedy's Rede. Obwohl die US-amerikanische Gesetzgebung sich in den letzten 50 Jahren weniger entwickelt hat als in Europa, so ist doch unlängst das Financial Protection Bureau etabliert und der freilich umstrittene - Dodd-Frank Wall Street Reform Act verabschiedet worden. Vom amerikanischen Vorbild können wir in Europa insbesondere auch über das Verhältnis der Bundesstaaten zur Föderation das Nötige lernen.

Anschließend erörterte Fabrizio Cafaggi (Europäisches Hochschulinstitut Florenz) die Frage, wie der Gesetzgeber den Verbraucher schützen solle: mit zwingendem Recht oder mit Informationspflichten. Auch wies er auf die Spannungen zwischen öffentlichrechtlichen und privatrechtlichen Schutzinstrumenten und auf die Unterschiede bei der Umsetzung der Richtlinienvorgaben in den europäischen Mitgliedstaaten hin. Leone Niglia (Universität Exeter) betonte in seinem Vortrag die verfassungsrechtlichen Aufgaben des Verbraucherrechts hinsichtlich der Effektivität in Europa. Sybe de Vries (Universität Utrecht) analysierte in seinem Vortrag die Spannungen zwischen Verbraucherschutz und Kartellrecht. Tjakie Naudé (Universität Kapstadt) warf die Frage auf, inwieweit der neue südafrikanische Consumer Protection Act auch nicht priviligierte Ver- 
braucher schützen kann. Das ist auch eine Frage, die im neuen europäischen Verbraucherschuzprogramm 2014-2020 angesprochen wird. Willem van Boom (Erasmus Universität Rotterdam) und Vanessa Mak (Universität Tilburg) hoben den Einfluss anderer wissenschaftlicher Disziplinen, insbesondere den des Behavioral economics, hervor. Mak betonte dies insbesondere mit Blick auf finanzielle Sachzusammenhänge, wo die financial literacy von Verbrauchern abhängig von Alter, Geschlecht, usw ganz unterschiedlich sein kann. Die abschließenden Beobachtungen von Ilona Wolfframvan Doorn (insbesondere über den Vorschlag für ein Gemeinsames Europäisches Kaufrecht) und Harrie Temmink (beide von der Europäischen Kommission) werden im Kon- ferenzbericht (Tijdschrift voor Consumentenrecht 2012, 94) gespiegelt.

Es versteht sich, dass nicht alle Fragen des Verbraucherrechts an einem Tag diskutiert werden können. Eher „technische" Fragen, wie die nach der Bestimmung des Verbraucherbegriffs - insbesondere, ob auch Minderkaufleute erfasst werden sollten sowie die Kodifikationsfrage (im BGB oder in Sondergesetzen; vgl dazu Micklitz, in Verhandlungen des 69. Deutschen Juristentages I A [2012]) - mussten außer Betracht bleiben. Die hier veröffentlichten Beiträge geben allerdings ein Bild von - wie Micklitz es in seinem Gutachten für den Deutschen Juristentag nennt - „der Dynamik" des Verbraucherrechts. 